\title{
Schaustellerei und Heilmittelvertrieb in der Antike
}

\author{
Von Fridolf Kudlien
}

Die hier zur Debatte stehende spezielle Form niederer Heiltätigkeit wurde erstmals von dem Altertumswissenschaftler Morel genauer erfaßt und dokumentiert ${ }^{1}$. Die moderne Medizingeschichtsschreibung hat für diesen Heilertyp, frühen Vorläufer des mittelalterlichen und späteren «Quacksalber» und «mountebank», vergleichsweise wenig Interesse gehabt. Im Folgenden wollen wir den von Morel beigebrachten Zeugnissen einige hinzufügen und die charakterisierenden Linien des Phänomens schärfer herausarbeiten, als es bisher (so etwa auch in meinen eigenen früheren Bemerkungen zum Thema ${ }^{2}$ ) geschehen ist.

Beginnen wir mit einem Zeugnis aus Galens Schrift «Über Antidote» (XIV 180 Kühn). Es handelt sich dabei um die Präsentation eines Gegenmittels gegen den Biß einer «phalangion» genannten Giftspinne. Als Urheber dieses Mittels wird Chariton «der 〈ochlagōgos»» angegeben. Dieser «zieht herum auf den Panegyreis, indem er den Gebissenen helfen will»(Galen a. O.). Auf S. 182 ebd. nennt Galen einen Simmias «ochlagōgos» mit einem ähnlichen, ebenfalls gegen «phalangion»-Biß «und den Biß jedes giftigen Reptils» wirkenden Mittel.

Hier sind zunächst zwei in unserem Zusammenhang besonders wichtige griechische Stichworte kurz zu erläutern. «ochlagōgos» meint jemanden, der die «Masse», den «Pöbel», «(ver)führt» oder «anzieht» (man denke an das sehr ähnliche «dëmagōgos»). Dies geschah am leichtesten anläßlich einer gut besuchten öffentlichen Veranstaltung. Die Panegyris, eine besonders stark frequentierte Festversammlung von ursprünglich religiösem Charakter, war sehr früh schon, ihrer Erscheinung nach, «ganz weltlich und profan» ${ }^{3}$; bei dieser Gelegenheit «konnte ein jeder auftreten, ... von den großen Geistern bis zu gemeinen Gauklern», und so schlugen hier etwa auch «Verkäufer Buden auf» (genauso wie sie das sonst auf Plätzen/Märkten taten).

Chariton war also offenbar ein «Marktschreier» im wahrsten Sinne des Wortes. Das (selten belegte) griechische Substantiv «ochlagōgos» führt uns nun zu einem entsprechenden lateinischen Terminus: Antike Glossarien erklären dieses Wort als «circulator» ${ }^{4}$ (und dieser Terminus erscheint auch, an der zitierten Galenstelle, in der der Kühnschen Ausgabe beigegebenen lateinischen Übersetzung). Unser Thema gibt uns Gelegenheit, auf einen das 
Verständnis des Wortes «circulator» bereichernden Umstand aufmerksam zu machen: «circulare» meint, in solchem Zusammenhang, sicher nicht bloß "to form circles or groups round oneself (for the purpose of making impromptu speeches, giving performances, etc.)". ${ }^{5}$ Sondern es ist zu beachten, daß das griechische Äquivalent von «circulus/circus», «kyklos», nach Auskunft der Wörterbücher, unter anderem den (mehr oder weniger kreisrunden) Platz/Markt bezeichnete. Auf solchen Plätzen/Märkten eben waren die «circulatores», ebenso wie die «ochlagōgoi» à la Chariton, normalerweise tätig. Ein anderer lateinischer Ausdruck, der ebenfalls in unseren speziellen Zusammenhang gehört, betont diesen Aspekt noch einmal explizit: «circumforaneus», zu «forum» gehörig (weiteres zu diesem Begriff nachher).

Der Fall des Chariton zeigt anschaulich, woher gegebenenfalls die Heilmittelkenntnis dieses besonderen Vertreters niederer Heiltätigkeit stammen mochte: Der Umgang mit solchen giftigen Tieren wie den «phalangia» gehörte zur Schaustellertätigkeit, wie sie auch die "pharmakopōlai» ausübten ${ }^{6}$. Als derartige Schausteller kannten solche Leute naturgemäß auch Mittel gegen Bisse von Giftspinnen oder -schlangen. Ebenso kannten sie Mittel etwa zur Betäubung von Giftschlangen, um dann mit diesen Tricks vorzuführen. Dies bezeugt Celsus De med. V27, 3 C («et si stupente ea [sc. colubra], quod per quaedam medicamenta circulatores faciunt, in os digitum quis indidit neque percussus est»). Daß derart erworbene Kenntnisse auch gefährlich wirkende Mittel betreffen konnten, schildert Cicero in seiner Rede «Pro Cluentio» 40 für einen «pharmacopola circumforaneus» namens L. Clodius aus Ancona: Dieser wurde durch eine hohe Geldsumme bestochen, Beihilfe zum Mord mittels einer tödlichen «potio» zu leisten; er machte sich danach rasch davon, da er ja ohnehin immer «eilig war, damit ihm viele Plätze/Märkte zur Verfügung standen». Aber auch Harmloses kam natürlich in Frage, wie etwa Hustenmittel, wozu Lukian («Apologia pro mercede conductis» 7) etwas Amüsantes beisteuert, wie es vergleichsweise jedem Marktschreier passieren könnte: «Du gleichst insgesamt jenem Pharmakopōles, der, als er ein Hustenmedikament anpries und versprach, es werde sofort den Leidenden Linderung schaffen, zwischendurch selber augenscheinlich von Hustenkrämpfen geschüttelt wurde.»

Wir haben bisher nur von Heilmittelanpreisung, -verkauf, -applikation gesprochen. Soweit solche Schausteller und Marktschreier mit dem Gebiet des Behandelns/Heilens befaßt waren, haben sie sich jedoch zumindest gelegentlich auf mehr als nur Medikamente kapriziert - manche haben auch Operationen, und zwar bemerkenswert unorthodoxe und sensationelle, 
ausgeführt ${ }^{7}$. Doch wollen wir hier weiterhin unser Hauptaugenmerk auf den Heilmittelvertrieb dieser Leute richten.

Zum erfolgreichen Anpreisen und Vertrieb von Heilmitteln auf Plätzen/Märkten oder größeren Veranstaltungen gehört eine beträchtliche verbale Durchschlagskraft. In einer Bezeichnung wie «Quacksalber» scheint auf diese Fähigkeit ausdrücklich abgehoben zu sein: In niederländ. «kwakzalver» steckt «kwakken» = prahlen ${ }^{8}$. Das Geschick, potentielle Kunden durch Reden zu überzeugen, heben denn auch antike Zeugnisse für unsere «pharmakopōlai» oder «circulatores» mehrfach hervor. Es handelt sich dabei um eine spezifische Suada, eine «vis dicendi rapida atque abundans» (Seneca Epist. 40,3) oder «extemporalis garrulitas» und «volubilitas orandi» (Quintilian Inst. orat. II 4,15 u. X 1). Der jüngere Plinius charakterisiert die Art, «wie ein circulator auf dem forum» redet, ironisch mit einem Demostheneszitat (Plin. Epist. IV 7,6): «Indem er die Stimme hebt und Frohsinn hineinlegt und aus voller Kehle schreit.» Diese Bemerkung deutet, wie wir meinen, keineswegs darauf hin, daß solche Marktschreier «auch wohl Vorträge oder Vorlesungen» hielten ${ }^{9}$, sondern soll offenbar nur die vulgärreißerische Art des Anpreisens charakterisieren.

Wie ernst war der Marktschreier, waren die von ihm angepriesenen Dinge - also etwa Heilmittel-zu nehmen? Besaß er, abgesehen von der Möglichkeit rein empirisch erworbener Kenntnisse (wie im Falle des Gegenmittels gegen Giftspinnenbiß), ein qualifiziertes, auf Ausbildung beruhendes Wissen? Daß dies nicht der Fall war, dürfte eine Petronstelle (Sat. 68) bezeugen, die zwar textlich nicht in Ordnung, dem Sinne nach jedoch durchaus verständlich ist: Von einem Sklaven, der bei einer Soiree eine geradezu scheußliche Vergilrezitation bot («nullus sonus unquam acidior percussit aures meas», bemerkt der Erzähler), erwähnt sein Herr stolz «er hat niemals gelernt, sondern ich schickte ihn zu den circulatores - er hörte zu» (daß das überlieferte «audibat» offenbar beizubehalten ist, wird sich nachher an einer durch eine Catostelle vermittelten Parallele erweisen). Das heißt doch wohl, daß die circulatores nicht als regelrechte Lehrer einer «technē/ars» zu nehmen sind, da ihre Tätigkeit nicht entsprechend zu klassifizieren ist und sie selber diese nicht regelrecht gelernt haben. Was sie als Marktschreier betreiben, ist also kein qualifizierter Beruf, sondern ein «Gewerbe» im ursprünglichen Sinne des mittelhochdeutschen Wortes, welches die Bedeutungen «wirbeln/kreisen, sich drehen; sich umtun, tätig sein; handeln; (an)werben» in sich schließt ${ }^{10}$. Dies mag sich seinerseits auch wiederum in Worten wie «circulator» oder «circumforaneus» mit abspiegeln. 
Soweit es um Heilmittel geht, war der Arzt als Professioneller, als Ausübender eines lehr- und erlernbaren, qualifizierten Berufes, der absolute Gegentyp zum Heilmittelvertreiber oder -applikateur, wie ihn der Marktschreier darstellt. So sah es auch die Antike, und zwar nicht nur die antiken Ärzte selber, von denen regelrechte Wutausbrüche gegen solche Marktschreier überliefert sind (Belege bei Morel a.0.), sondern so empfanden es auch Laien. Wenn der hellenistische Popularphilosoph Teles die Möglichkeit erwägt, in einer Polis könnten die dafür Zuständigen «den qualifiziertesten Arzt wegschicken, einen Pharmakopolēs wählen und diesem die öffentliche Aufgabe übergeben», ${ }^{11}$ so ist das nur ein hypothetischer Fall - vorgekommen wird dies bestimmt nie sein. Vom alten Cato, dem Censor, gibt es hierzu eine besonders treffende, wörtlich überlieferte Äußerung (s.Gellius Noct. Att. I 15,9): «Ihr hört zu (auditis), hört (aber) nicht darauf (auscultatis), wie einem 〈pharmacopolas. Denn dessen Worte werden angehört, in Wirklichkeit aber überläßt sich ihm im Krankheitsfall niemand». Hier zeigt die sorgfältige Unterscheidung zwischen bloßem «audire» und aufnehmendbefolgendem «auscultare» sehr schön, worauf es jeweils beim Heilmittel vertreibenden Marktschreier und beim professionellen Arzt ankommt (eben wegen dieser sprachlichen Unterscheidung möchte ich an der oben vorgestellten Petronstelle das überlieferte, dort offenbar genau passende «audibat» beibehalten sehen). Ob nun allerdings Cato rechtgehabt hat mit der Behauptung, niemand höre in Wirklichkeit auf den Marktschreier und seine Heilmittel-Anpreisungen, muß doch sehr dahingestellt bleiben - der Scharlatan besitzt, wie auch wir zur Genüge wissen, seine eigene Anziehungskraft, und die vielen antiken Attacken gegen ihn zeigen in ihrer geballten Heftigkeit deutlich genug, daß er keineswegs eine quantité négligeable war, daß er vielmehr seine Kunden fand, daß also Catos Bemerkung eher als mahnender Appell denn als Tatsachenfeststellung zu nehmen ist.

Mit «Scharlatan» ist unversehens ein nur scheinbar unproblematisches, in der Tat hier eher irreführendes Stichwort gefallen. Zwar signalisiert die romanische Grundbedeutung des Wortes ebenfalls den «Schwätzer, Marktschreier». Aber: Auch der professionelle Arzt kann gegebenenfalls ein Scharlatan sein und so genannt werden. Sind jedoch unter den antiken Marktschreiern, wie wir sie hier, für den Bereich des Heilmittelvertriebs, philologisch-historisch unter die Lupe nehmen, auch professionelle Ärzte vorstellbar? Man hat z. B. den Chariton, mit dem wir unseren Überblick begannen, schlankweg als «W anderarzt» aufgefaßt ${ }^{12}$. Dies müssen wir, nach unseren Ausführungen, für falsch halten. Warum sollte denn Galen für jenen 
in einem jeglicher Polemik entbehrenden Zusammenhang die Tätigkeitsbezeichnung «ochlagōgos» verwenden - wenn nicht, um damit deutlich zu machen, daß es sich eben nicht um einen Arzt, sondern um einen echten Schausteller/Marktschreier, einen wirklich das Gewerbe eines «circulator» Betreibenden handelte?

Davon streng zu unterscheiden sind, wie gesagt, Ärzte, welche scharlatan-hafte Züge aufweisen. In seiner berühmten «Sittengeschichte» hat Friedländer ein paar Belege für die römische Kaiserzeit gegeben ${ }^{13}$. Aber schon im Corpus Hippocraticum wird dieser Arzt-Typ mit Ausdrücken kritisiert, die wir als den echten Marktschreier charakterisierende kennengelernt haben: In Hipp. Artic. 42 u. 44 (Littrés Zählung), einer alten Schrift, heißt es von Ärzten, welche eine bestimmte knochenchirurgische Therapiemethode anwenden, daß sie «bestrebt sind, die große Masse (〈ochlos〉) einzuseifen»; in jedem qualifizierten Beruf, und besonders im ärztlichen, sei «viel Gedränge (〈ochlos〉), viel Schau (〈opsis〉), viel Marktschreierei (〈logos〉)» von Übel - dies gezieme eher den «Gauklern» (〈apateōnes»). Und in der hellenistischen, deontologischen Schrift «Peri euschēmosynēs» Kap.2 ist wieder, diesmal generell, ablehnend von all jenen die Rede, die «einen Volksauflauf hervorrufen, durch Gaukelei (〈apateontes〉), und indem sie in den Poleis als 〈circulatores〉 herumziehen». Die letzten Worte unserer Übersetzung beziehen sich auf den griechischen Ausdruck «anakykleontes»; es scheint noch nicht bemerkt zu sein, daß dieses Verbum, in solchem Zusammenhang (es hat auch andere Bedeutungen), das perfekte Äquivalent zu lat. «circulare» darstellt. Obwohl dieser hellenistisch-ärztliche Verfasser als Gegenbild dazu die wahre «technē» hinstellt, die sich dadurch auszeichne, daß sie nach einer «methodos technikē», einer qualifizierten Methodik, praktiziert werde (ebd.), hat auch diese Schrift offenbar, wie die vorher zitierte knochenchirurgische, mit den «Gauklern/Marktschreiern» nicht wirkliche «circulatores», sondern Vertreter qualifizierter Berufe im Sinn, die es wie jene treiben. Dieser Kontext einer kritischen Unterscheidung innerhalb des «technē»-Bereichs, zwischen wahren und dubiosen «technitai», speziell: seriösen und scharlatanhaften Ärzten, unterscheidet die genannten hippokratischen Zeugnisse von Galens Nachricht über Chariton, wo es sich um einen echten «circulator» handelt, und von all den anderen Nachrichten, die sich mit wirklichen «pharmakopōlai» und ihrem Gewerbe befassen.

Eine interessante Frage wäre nun die nach den eigentlichen Gründen, warum ein professioneller Arzt sich wie ein Marktschreier/Schausteller 
benahm. War gegebenenfalls dabei Überkompensation einer mangelhaften Qualifikation im Spiel? Sah der Betreffende darin das wirksamste Mittel, möglichst viele Patienten zu bekommen? Ebenso interessant wäre die Komplementärfrage, wie dann die standesethisch strengen, jeden Anstrich von Scharlatanerie bekämpfenden Ärzte ihrerseits an Patienten kamen, den Konkurrenzkampf erfolgreich bestanden - allein durch Untadeligkeit? Nach welchen Kriterien eigentlich bemaß sich der «gute Ruf» eines Arztes im Publikum? Doch so reizvoll solche Fragen sind: Wir haben es hier nun einmal nicht mit den regelrechten Ärzten, sondern mit einer bestimmten Form «niederen» Heilgewerbes zu tun.

Das Stichwort «Gaukler», das uns anläßlich des griechischen «apateōn» ebenso leicht wie der Begriff «Scharlatan» in die Feder kam, bedarf ebenso wie dieser einiger Modifikation. Gewiß «gaukelte» der Heilmittel anpreisende, verkaufende, verabreichende «circulator» seinem Publikum oft etwas vor (s. den oben erwähnten Fall des Hustenmittels). Heftet man nun aber das deutsche Wort an die verschiedenen in Frage kommenden antiken Termini, so muß man vorsichtig mit einem Definitionsversuch sein: Wenn man dabei den Begriff «circulator» mit hineinnimmt, dann darf man den derart verstandenen «Gaukler» nicht lediglich als «Belustiger»- mit der Unterteilung in «Artist» oder «Zauberer»-definieren ${ }^{14}$. Der Umgang mit Heilmitteln gehört eben gegebenenfalls auch dazu (und er ist nicht abgedeckt durch solche einengende Festlegung). Für unsere Zwecke schien uns, nach allem, der Begriff «Schausteller» mit seinem breiten, den Warenvertrieb mit einschließenden Wortfeld am geeignetsten zu sein.

Wir erwähnten zu Anfang, daß moderne Medizingeschichtsschreibung den Heilmittel vertreibenden Schausteller der Antike wenig beachtet hat. Das gleiche gilt für moderne Erforscher antiker Märkte (ohnehin ein noch kaum richtig in Angriff genommenes Forschungsgebiet ${ }^{15}$ ) - obwohl doch auch die «circulatores» ein Gewerbe betrieben, das sich, wie wir sahen, vor allem auf Plätzen/Märkten abspielte ${ }^{16}$. Bedenkt man die Fülle der Märkte allein in den Städten (von Dörfern und gänzlich isolierten ländlichen Märkten ganz abgesehen) und die sich häufenden Markttage, was beides in noch erhaltenen antiken Marktkalendern sich spiegelt ${ }^{17}$, so wird unmittelbar anschaulich, warum der pharmacopola L. Clodius immer «in Eile war» (s.o.).

Welche Stellung nahm der unter anderem Heilmittel vertreibende Schausteller/Marktschreier in der antiken Gesellschaft ein? Gehörte er zu den «Unterschichten», deren Erforschung heute ein besonderes Anliegen der 
Sozialhistoriker ist? Der Begriff «Unterschichten» erweist sich bei kritischer Prüfung selber als so vage und dehnbar, daß man schon deshalb bei der Beantwortung der eben gestellten Frage vorsichtig sein muß. Man darf sich dabei vor allem nicht durch die vielen, den uns hier interessierenden Gewerbetyp scharf abwertenden antiken Zeugnisse einseitig beeinflussen lassen. Solche Urteile repräsentieren ja tatsächlich nur eine Seite der Sache. Daß etwa Horaz (selber, in der Sicht der «Oberschicht» sensu stricto, von eher zweifelhafter gesellschaftlicher Herkunft) die "pharmacopolae» ironisch abwertet, indem er sie gemeinsam mit Dirnen, (religiösen) Bettlern und Possenreißern als «hoc genus omne» einstuft (Sat. I 2,1 f.), ist ebenso vom Psychologischen wie vom Künstlerischen her (Eigenarten des literarischen Genre «Satire») zu sehen. Auf der anderen Seite darf man nicht außer acht lassen, daß mindestens einige «pharmakopōlai» Diener hatten, die dann ihrerseits sozial unter ihren Herren standen (sie waren oft Sklaven, s. Morel a. O.). Auch konnten die Vertreter dieses Gewerbes in Rom «collegia» mit der qualifizierenden Bezeichnung «öffentlich» bilden ${ }^{18}$, was auf einen gewissen gesellschaftlichen Anspruch, aber eben wohl auch auf eine gewisse gesellschaftliche Geltung hindeutet. Und schließlich ist durchaus nicht auszuschließen, daß ein "pharmacopola circumforaneus» wie L. Clodius aus Ancona ein ingenuus (freigeborener Römer) gewesen ist. Auch Chariton (welcher der Liste namentlich bekannter Schausteller/Marktschreier bei Morel a.O. hinzuzufügen wäre) mag, als Nicht-Römer, der er offenbar gewesen ist, durchaus in die Kategorie der Freigeborenen (Peregrine?) gehört haben, ebenso wie andere in Morels Liste. Das gleiche wie für Chariton gilt für Simmias. Für ihn gibt Galen a. O. S.180 Z.10 die Filiation («tou Mēdiou» = Sohn des Medios) an, was ein zusätzlicher Hinweis auf den Freigeborenen-Status sein kann.

Nimmt man dies alles mit der Tatsache zusammen, daß die uns hier beschäftigenden Leute zweifellos ein recht bekanntes, verbreitetes, seiner Kundschaft sicheres Gewerbe, aber eines ohne signifikant «schmutzigen» Anstrich (wie etwa das der Lastträger, der Walker o. ä. eines war), ausübten, dann wird man sie sicher nicht korporativ an der allertiefsten Stelle der gesellschaftlichen Pyramide der Antike plazieren wollen. 


\section{Anmerkungen}

1 W. Morel, Art. «Pharmakopoles», Pauly-Wissowa RE XIX 2, 1938, Sp. 1840 f.

${ }^{2}$ F. Kudlien, Der griechische Arzt im Zeitalter des Hellenismus, Wiesbaden 1979 (Abh. Ak. Mainz 1979, 6), S.94f.

${ }^{3}$ Dieses und die folgenden Zitate stammen von M.P. Nilsson, Geschichte der griechischen Religion, Bd.1, München $1967^{3}$, S. $826 \mathrm{f}$.

${ }^{4}$ s. Liddell-Scott-Jones, A Greek-English Lexicon, s.v. «ochlagōgeus».

${ }^{5}$ So das Oxford Latin Dictionary s.v.

${ }^{6}$ Dazu s. Morel a. O.

7 Dazu s. meinen Beitrag «Diodors Zwitter-Exkurs als Testimonium hellenistischer Medizin», Clio Medica 1, 1966, S.319-324.

${ }^{8}$ s. Duden Bd.5, Ausg. 1980, s.v.

9 So Mau, Art. «Circulator 1», Pauly-Wissowa RE III 2, 1899, Sp. 2570.

${ }^{10}$ Vgl. Duden Bd. 3, 1977, s.v.

11 Teles p. $18,10 \mathrm{f}$. ed. Hense.

12 M. Wellmann, Art. «Chariton 4», Pauly-Wissowa RE III 2, 1899, Sp. 2171.

${ }^{13}$ L.Friedländer, Darstellungen aus der Sittengeschichte Roms, Bd.1, 10. Aufl., Nachdr. 1977, S. $200 \mathrm{f}$.

${ }^{14}$ So C. Zintzen, Art. «Gaukler», Der Kleine Pauly, 11. Lief., 1966, Sp. 703.

${ }^{15}$ Vgl. R.MacMullen, Markttage im Römischen Imperium, in: H. Schneider (Hrsg.), Sozialund Wirtschaftsgeschichte der Römischen Kaiserzeit, Darmstadt 1981, S. 280-292.

${ }^{16}$ Ebd. S. 284 u. ö. ist übrigens die «panegyris» (vgl. o.S.91) als «Markt» erwähnt.

17 Vgl. dazu ebd. S. $290 \mathrm{f}$.

${ }^{18}$ Corp. Inscr. Lat. V 4489.

\section{Summary}

This essay is concerned with the Graeco-Roman "mounte-bank" acting as a drug-vendor. Particular emphasis has been laid upon the explanation of related terms (such as 'ochlagōgos' or 'circulator'). As far as possible, we have tried to define the place of this lower (nonprofessional) type of 'healer' in Graeco-Roman society and to bring him to life, so to speak.

Prof. Dr. F. Kudlien

Goethestraße 3

D-2300 Kiel 